\title{
Indian Subcontinent's First Bilateral Supracondylar Level Upper Limb Transplantation
}

\author{
Mohit Sharma ${ }^{1} \quad$ Subramania lyer ${ }^{1} \quad$ Kishore P. $^{1} \quad$ Jimmy Mathew ${ }^{1} \quad$ Janarthanan R. ${ }^{1} \quad$ Nirav Maharaja \\ Swapnil Dhake Akshay Omkumar $^{1}$ Thomas Joe ${ }^{1} \quad$ Dimpy Sharma $^{1} \quad$ Radhika Kapathia $^{1}$ Ankita Harijee ${ }^{1}$ \\ Srilekha Reddy ${ }^{1}$ Jerry Paul ${ }^{2}$ Sunil Rajan ${ }^{2}$ George Kurian ${ }^{3}$ Zacharia Paul ${ }^{3}$ Druvan Shaji ${ }^{4}$ \\ Balu C. Babu ${ }^{4}$ Anubhav Sharma ${ }^{4}$
}

\footnotetext{
${ }^{1}$ Department of Plastic and Reconstructive Surgery; Amrita Institute of Medical Science, Kochi, Kerala, India

${ }^{2}$ Department of Anaesthesiology, AIMS Kochi; Amrita Institute of Medical Science, Kochi, Kerala, India

${ }^{3}$ Department of Nephrology, AIMS Kochi; Amrita Institute of Medical Science, Kochi, Kerala, India

${ }^{4}$ Department of Orthopedics, AIMS Kochi; Amrita Institute

of Medical Science, Kochi, Kerala, India
}

Address for correspondence Dr. Mohit Sharma, MS, MCh, Professor and Head of the Department of Plastic and Reconstructive Surgery, Amrita Institute of Medical Sciences, Kochi 682041, Kerala (email: ammasmohit@gmail.com)

\begin{abstract}
Keywords

- bilateral hand transplantation

- hand transplantation

- Indian hand transplantation

- supracondylar hand transplantation

- vascularized tissue allotransplantation

Introduction This is the first case of supracondylar level transplant from the Indian subcontinent, performed for a bilateral below elbow amputee. It has a completely different set of challenges for the transplant team, with a relatively shorter ischemia time window.

The technical considerations for the same have been discussed in detail in this article.

Materials and Methods The patient was a 19-year-old female who lost her both upper limbs at proximal forearm level due to severe crush injury following a road traffic accident. Insufficient bone length on either side necessitated a supracondylar level transplant. The preoperative workup included detailed clinical evaluation, biochemical, and psychological evaluation.

The donor was a young brain-dead, male patient from a hospital, 30 minutes away. The donor and recipient preparations in this case were unique. The recipient's own elbow flexors and extensors were used while the elbow joint was from the donor. The specific challenges we faced during this procedure have been described in detail.

Results The transplantation has been a complete technical success, with the patient rehabilitated back to her independent life style. This article describes only the technical considerations. The functional recovery aspect is part of an another soon to be published manuscript.

Conclusion Supracondylar level arm-transplant requires a highly coordinated team effort with precise preoperative planning, along with meticulous attention to detail to achieve a successful outcome. In properly selected patients, it could be a life-changing procedure, worth all the effort.
\end{abstract}

\section{Introduction}

Vascularized composite allotransplantation (VCA), a surgical procedure that has evolved from close collaborations

received

July 16, 2019 accepted after revision October 14, 2019

published online

December 26, 2019
DOI https://doi.org/

10.1055/s-0039-3401466

ISSN 0970-0358. between reconstructive and transplant surgeons, has opened a new horizon in the field of reconstructive surgery. The known VCAs being performed across the world are hand, face, uterus, penile, abdominal wall, larynx, and knee. ${ }^{1,2}$ Hand
(C)2019 Association of Plastic

Surgeons of India
License terms

(요 (1) $\Theta \circledast$ 
transplantation is the most commonly performed VCA across the world with close to 120 cases of upper limbs being transplanted as per "The International Registry on Hand and Composite Tissue Allotransplantation."3

Since 1998, when the first successful hand transplant was performed but resulted in an amputation after a period of 2 years, the transplant immunology has come a long way with breakthroughs that have changed the future of reconstructive transplant surgery. ${ }^{4}$ The challenge of immunosuppression in the VCA is unique in a way that there are multiple tissue types included in the graft with varying immunogenic properties. $^{4}$

Upper arm-level or transhumeral transplantations are performed less frequently than forearm and hand transplantations for the following reasons:

1. The great distance of nerve regeneration required to reinnervate a large number of muscles, mainly the intrinsic musculature of the hand.

2. Large amount of muscle mass present in the transplant, which demands shorter periods of ischemia for adequate functional return and carries the risk of greater complications, including death.

3. Unpredictable extrinsic innervation and sensory recovery, with no predictable hand intrinsic muscle reinnervation. ${ }^{5}$

Therefore, the patient requires intense and prolonged rehabilitation, as well as a greater multidisciplinary support to obtain basic function of the limb. ${ }^{6}$ As a result of these concerns, identifying appropriate patients for this procedure has been more difficult than for the more "routine" distal forearm hand transplant patients.

The first report of an arm-level transplantation was published in Germany by Höhnke et al. The transplant was distal to the insertion of the deltoid muscle and had only one functional report with a follow-up of 2 years. ${ }^{7}$ Till date around 10 arm-level transplantations have been reported. The most proximal level at which an upper limb transplant has been performed was reported by Martin Iglesias in 2015 where one limb was transplanted at the level of glenohumeral joint and other at midarm level. ${ }^{7-10}$

In the Indian subcontinent, however, this is a relatively new endeavor, with only a handful of centers having approval for performing the procedure. This case is the fourth in the country to undergo hand transplantation.

The above elbow amputees may suffer from significantly higher nerve avulsion injuries than would be expected based on their limb amputation level. To define the level of nerve injury, a high-resolution magnetic resonance neurography is performed in addition to detailed physical examination. ${ }^{11}$

In this article, we describe the surgical technique for the supracondylar level upper limb transplantation.

\section{Case Details}

A 19-year-old female patient sustained crush injury of both her upper limbs following a road traffic accident, for which she underwent bilateral below elbow amputation (-Fig. 1) showing preoperative photograph of the patient). Her disabilities of the arm, shoulder, and hand (DASH) score was 68.9. The patient was otherwise healthy, well-motivated and was not having any psychological impairments as determined after a thorough clinical evaluation. She had good family support who understood the risks and consequences of the procedure well and was financially sound. Hence, she was considered as an ideal candidate for upper limb transplantation.

Bilateral upper limb transplantation was performed on 9th August 2017. Both the limbs were transplanted at the supracondylar level. The multiorgan donor was a 20-yearold male patient with brain death secondary to severe head injury following a road traffic accident.

The details of the preoperative evaluation performed for this case are mentioned in - Table $\mathbf{1}$. The donor had been admitted in a hospital that was 30 minutes away from our center.

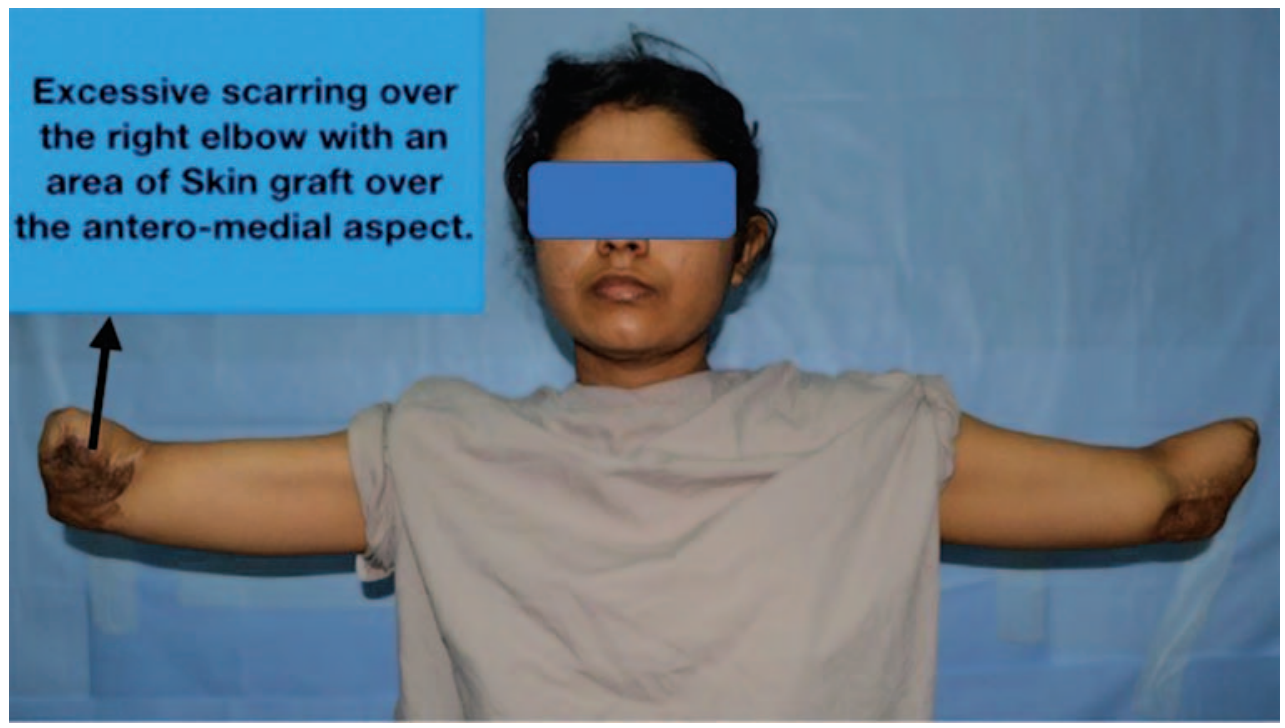

Fig. 1 Preoperative photograph of the patient. 
Table 1 Details of preoperative evaluation

\begin{tabular}{|c|c|c|}
\hline Details & Recipient & Donor \\
\hline Age & $19 y$ & $20 y$ \\
\hline Gender & Female & Male \\
\hline Blood group & $A+$ & $A+$ \\
\hline \multicolumn{3}{|l|}{ Serology } \\
\hline CMV & + ve & - ve \\
\hline Rubella & $+\mathrm{ve}$ & $+\mathrm{ve}$ \\
\hline Toxoplasmosis & $-v e$ & -ve \\
\hline EBV & $+v e$ & $+v e$ \\
\hline Lymphocyte crossmatch & \multicolumn{2}{|c|}{ Favorable (<10\%) } \\
\hline PRA & \multicolumn{2}{|c|}{ Negative for class I and class II } \\
\hline HLA & \multicolumn{2}{|c|}{$\begin{array}{l}\text { Typing could be done only for the } \\
\text { recipient }\end{array}$} \\
\hline
\end{tabular}

Abbreviations: CMV, cytomegalovirus; EBV, Epstein-Barr virus; HLA, human leucocyte antigen; PRA, panel reactive antibody.

\section{Patient Preparation and Technical Details}

1. Immunosuppression induction therapy:

- Day 0 (on the day of surgery)

Antithymocyte globulin (ATG) $75 \mathrm{mg}(1.5 \mathrm{mg} / \mathrm{kg})$ intravenous infusion.

Methyl prednisolone (MP) $500 \mathrm{mg}$ stat in $100 \mathrm{~mL}$ saline intravenous infusion.

Mycophenolate mofetil (MMF) 500 mg per-oral.

Tacrolimus $3.5 \mathrm{mg}$ BD per-oral.

Just before clamp release-500 mg intravenous MP.

Immediately after clamp release-500 mg MP intravenously.

- Day 1:

MP 250mg intra-venous.

ATG $50 \mathrm{mg}$ intra-venous.

Ischemia time:

Right side: Warm ischemia-10 minutes; cold ischemia-5 hours: 20 minutes

Left side: Warm ischemia-10 minutes; cold ischemia-5 hours: 20 minutes

2. Operative Details:

\section{Recipient Preparation:}

Under tourniquet control, midlateral incision was given on both sides extending from the stump up to the proximal arm (-Fig. 2) showing midlateral incision on the right arm and (-Fig. 3) showing midlateral incision on the left arm). Anterior and posterior skin flaps were raised deep to the deep fascia. The point where the cutaneous nerves and the cephalic and basilic veins were identified, the deep fascia was incised and the cutaneous nerves as well as the superficial veins were dissected off the skin for some distance. This step helps in aligning the structures in the straight line at the time of anastomosis, prevents kinking of vessels, and also facilitates the trimming of skin flaps at the time of final closure.

A systematic dissection plan was adopted and structures were identified, dissected, and tagged from radial to ulnar

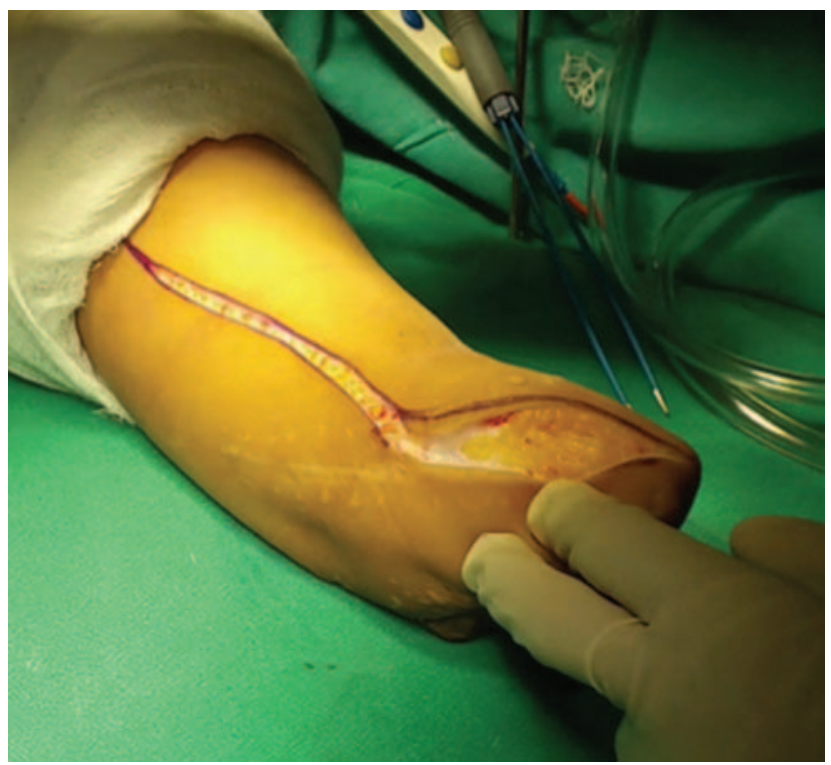

Fig. 2 Midlateral incision on the right arm.

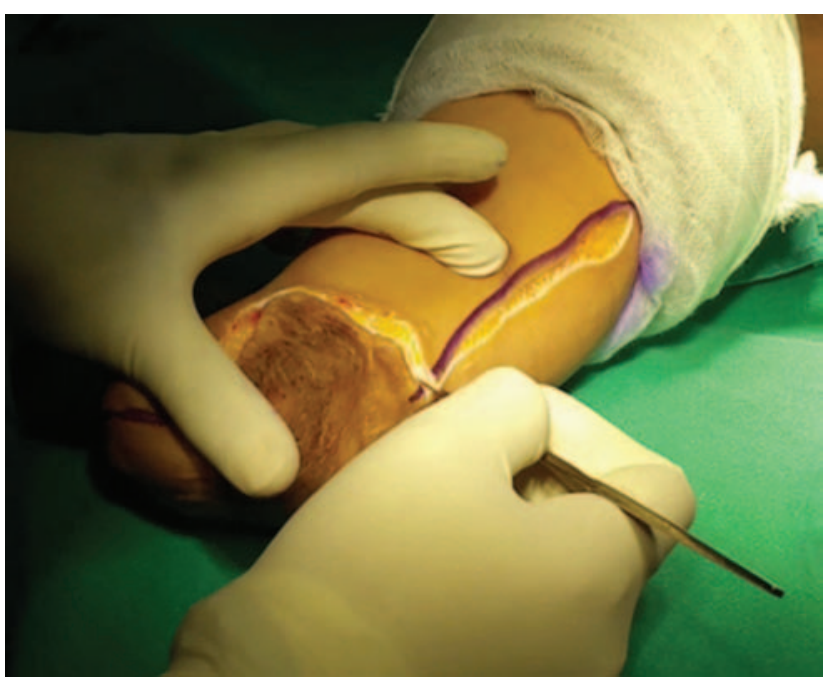

Fig. 3 Midlateral incision on the left arm.

side. The radial nerve was identified between brachioradialis and brachialis in arm and followed distally, identifying the superficial and deep branches, which were followed till the stump (-Fig. 4) showing dissection of the radial nerve). The branches of radial nerve to the brachioradialis, extensor carpi radialis longus (ECRL), and brevis were identified, transected, and tagged individually. Brachioradialis muscle belly that was intact on the left side was dissected along with its motor nerve.

On the right side, the radial nerve was encased in thick scar tissue; therefore, $10 \mathrm{~cm}$ of unhealthy nerve was excised along with the neuroma. On the left side, the radial nerve was mostly healthy and only the distal neuroma was excised. Similarly, the median and ulnar nerves were identified and dissected up till the elbow crease and the distal neuromas were excised. The right-sided median nerve also had a neuroma $10 \mathrm{~cm}$ above the distal most end and as a result that segment of nerve was also excised (-Fig. 5) showing the dissection of the ulnar nerve (yellow arrow) 


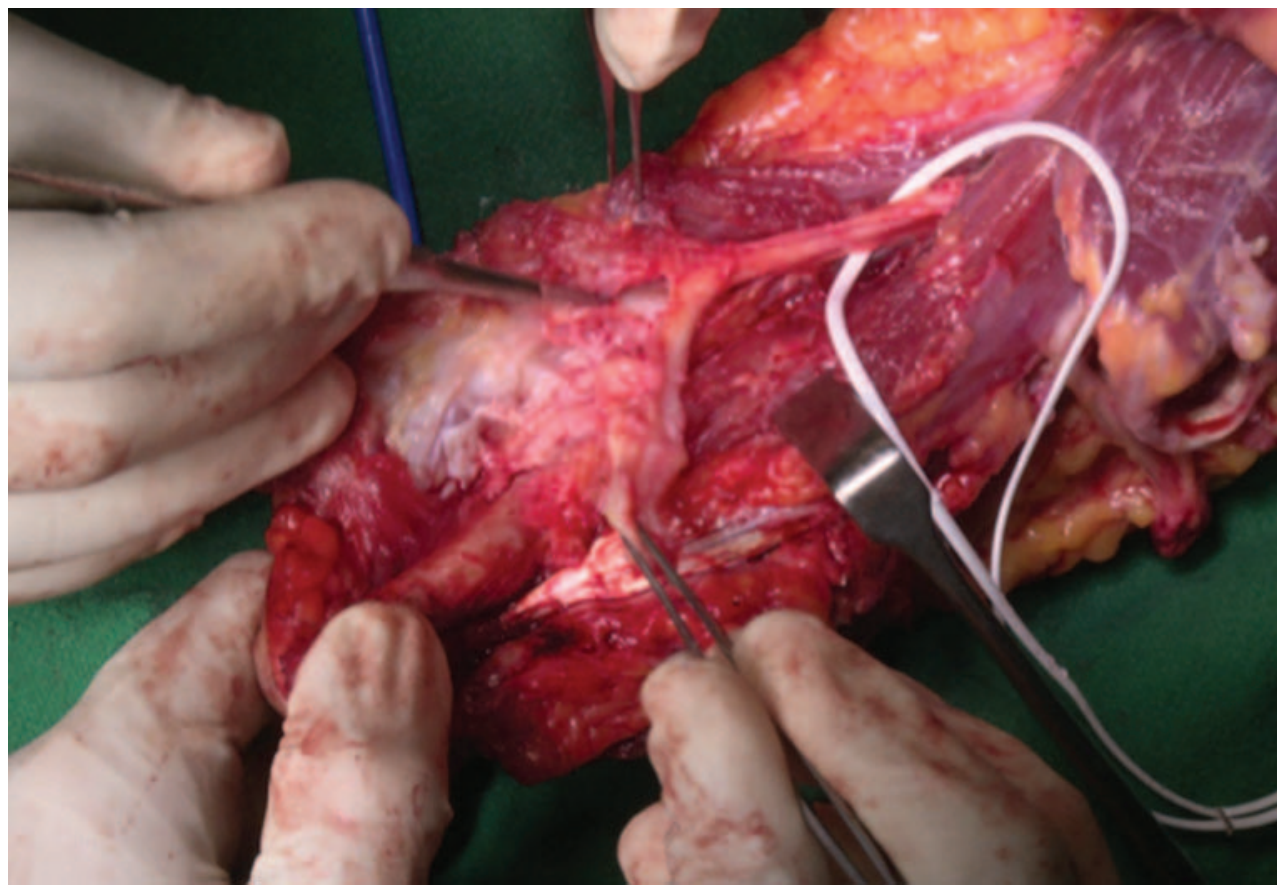

Fig. 4 Dissection of the radial nerve.

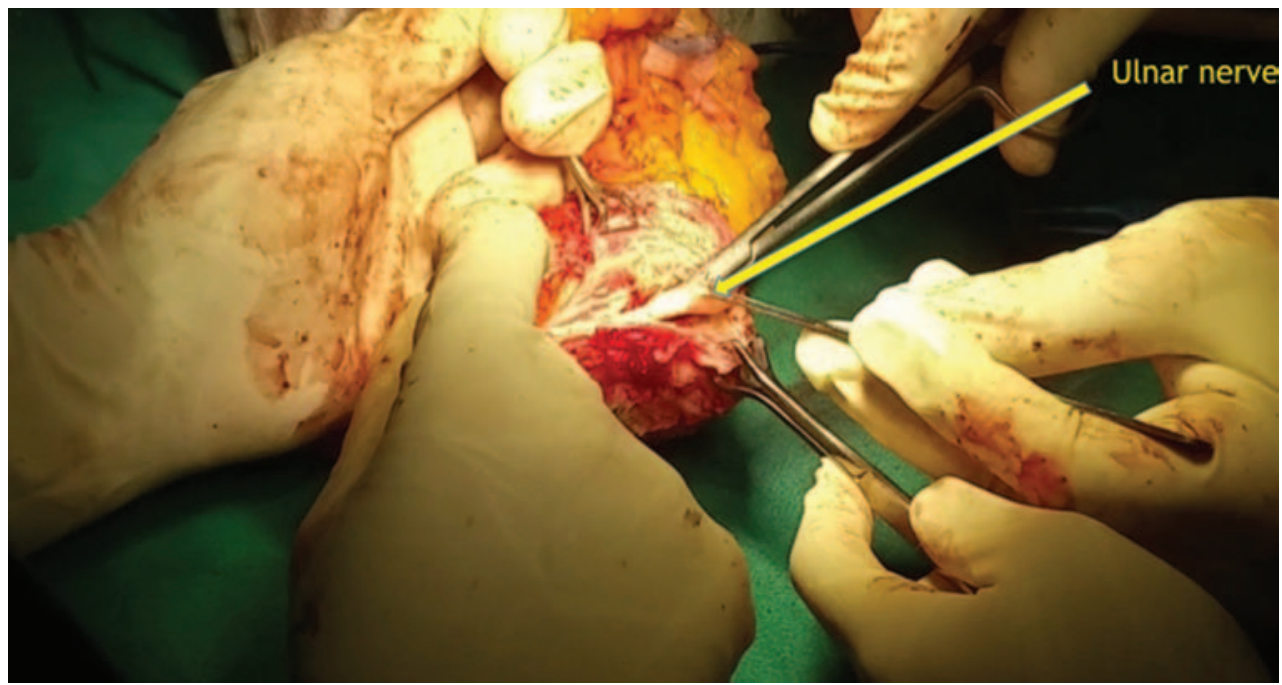

Fig. 5 Dissection of the ulnar nerve (yellow arrow).

and - Fig. 6 (blue arrow) showing neuroma in the median nerve). The lateral and medial cutaneous nerve of forearm was also identified and tagged on either side ( $\mathbf{- F i g . ~ 7 )}$ showing dissection of medial antebrachial cutaneous nerve and ( $\mathbf{- F i g . ~ 8 ) ~ s h o w i n g ~ d i s s e c t i o n ~ o f ~ l a t e r a l ~ a n t e b r a c h i a l ~}$ cutaneous nerve).

Biceps brachii, brachialis, and triceps were raised along with their tendinous attachment and dissected up to the supracondylar region (- Fig. 9) showing biceps brachii being dissected and (-Fig. 10) showing brachialis muscle being dissected). Brachial artery along with its venae commitantes was dissected up till just above the elbow crease; each structure was individually tagged.

Humerus was exposed circumferentially and a proximally based periosteal flap was raised up to a length of $4 \mathrm{~cm}$ from the planned osteotomy site; this was done to cover and reinforce the site of osteosynthesis. The lateral epicondyle was exposed and length of the donor bone required to accommodate four holes of the $4.5 \mathrm{~mm}$ limited contact dynamic compression plate (LCDCP) was determined; in this case it was $8.5 \mathrm{~cm}$ proximal to the lateral epicondyle. This means that $8.5 \mathrm{~cm}$ of recipient humerus needed to be discarded for accommodating four holes of the dynamic compression plate on to the donor humerus, after this marking the remaining length of the bone was discarded, performing an osteotomy with an oscillating saw.

Further space was created on the lateral border of the recipient humerus by dissecting the lateral head of triceps and brachialis away, to accommodate the remaining four holes of the LCDCP. 

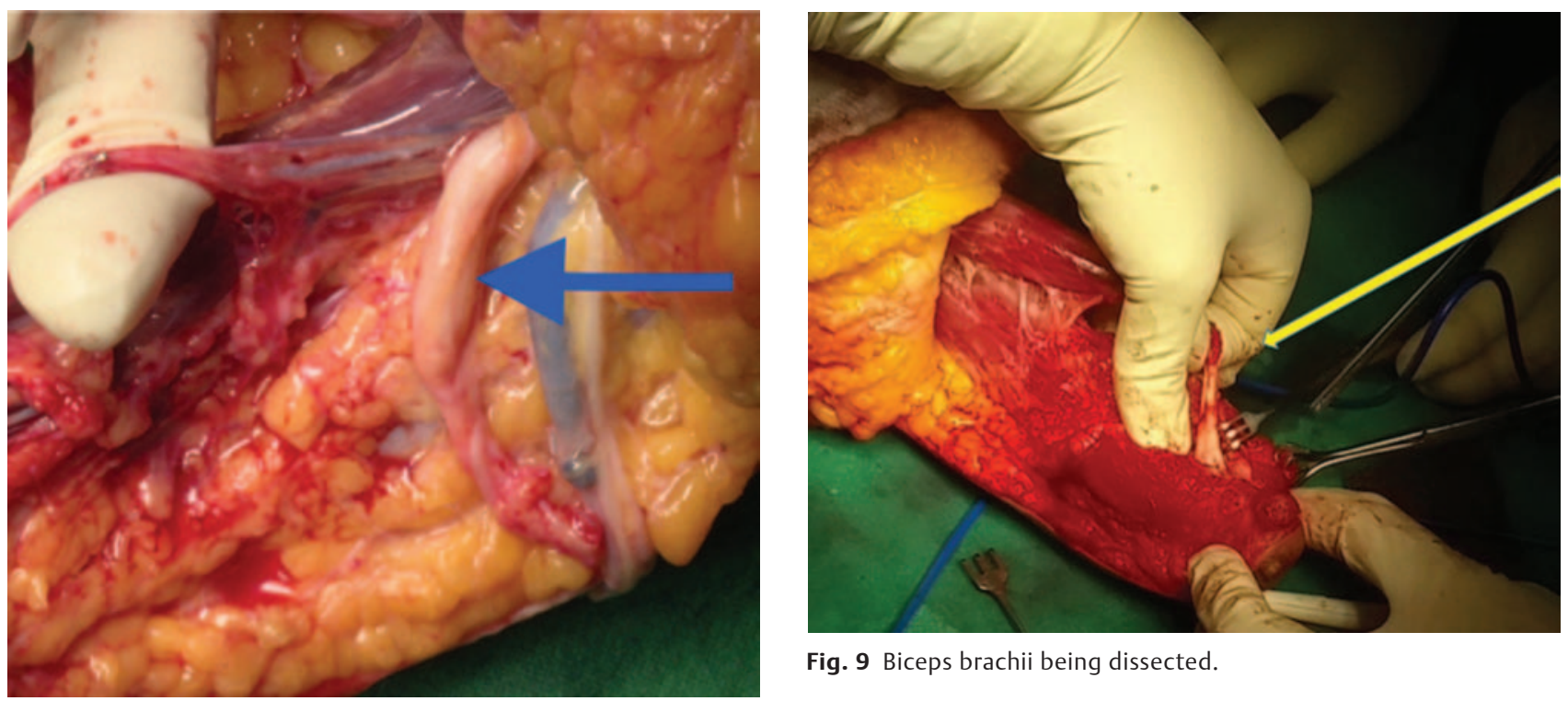

Fig. 9 Biceps brachii being dissected.

Fig. 6 Neuroma in the median nerve (blue arrow).

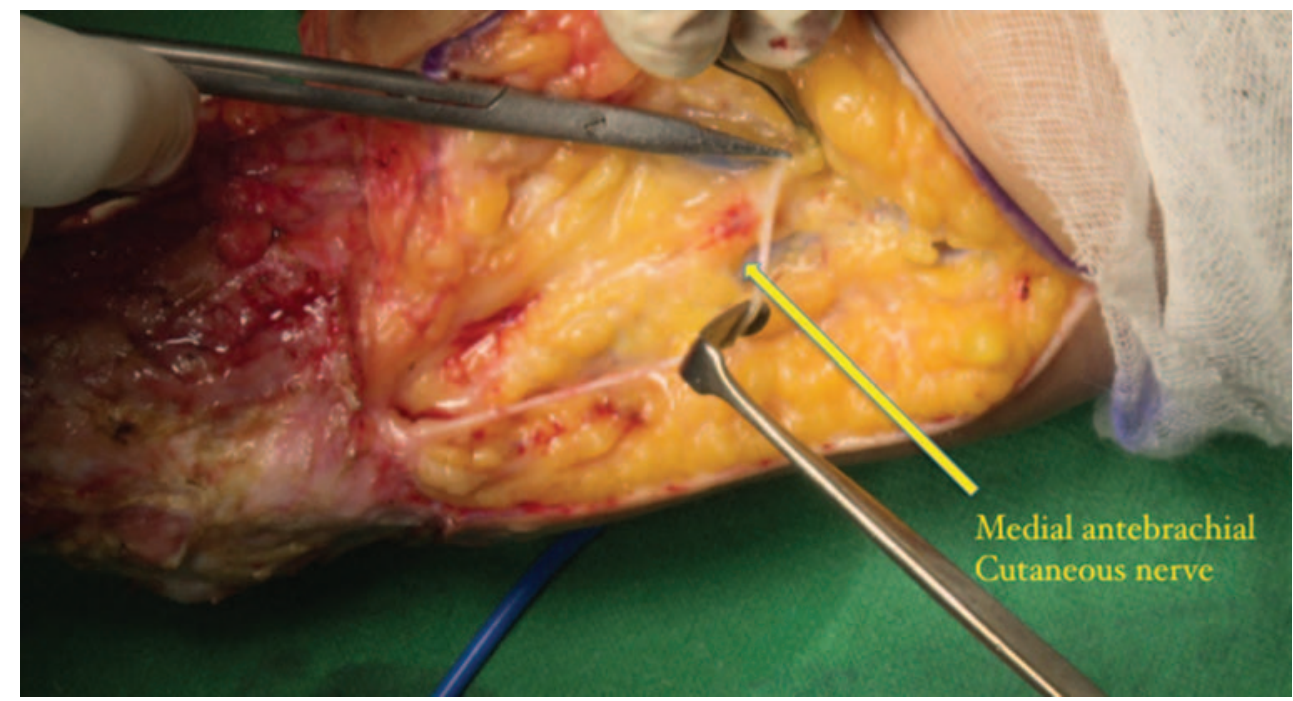

Fig. 7 Dissection of medial ante-brachial cutaneous nerve.
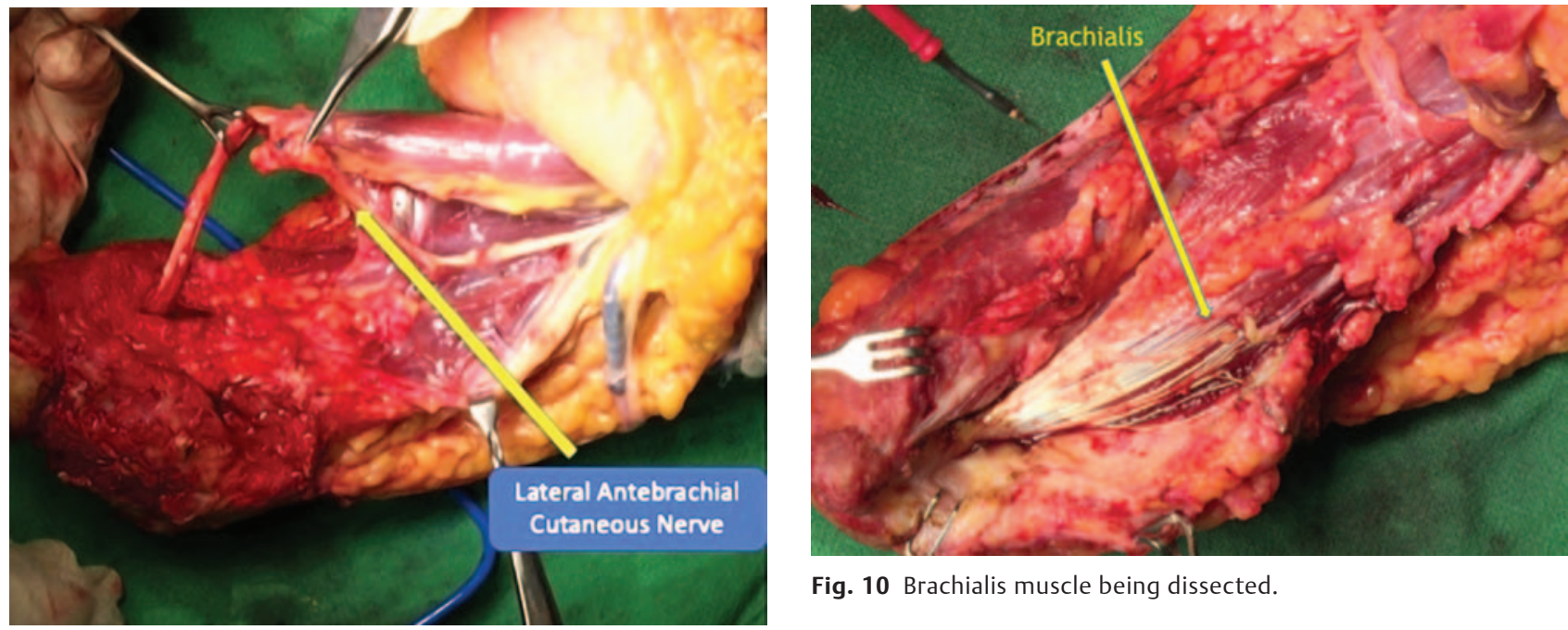

Fig. 10 Brachialis muscle being dissected.

Fig. 8 Dissection of lateral ante-brachial cutaneous nerve. 
Throughout the course of this dissection, the radial nerve trunk was identified and protected. A single $4.5 \mathrm{~mm}$ LCDCP with four holes was fixed on to the recipient humerus with bicortical screws.

\section{Donor Limb Retrieval}

A nonpneumatic tourniquet was used at the proximal arm; circumferential skin incision was given at midarm level (-Fig. 11) showing the retrieved donor upper limbs); all major superficial veins were clipped proximally and transected; all the muscles and major nerves were transected; the brachial artery was identified and clipped proximally and transected and osteotomy of the humerus was done at midarm level; immediately thereafter the limb was perfused with $1 \mathrm{~L}$ of University of Wisconsin solution and the velocity of flow was noted from the veins; in this case the flow was higher from the venae commitantes, which signified that it would be prudent to do the anastomosis of venae commitantes before the superficial venous anastomosis.

This simple observation helped in prioritizing the anastomotic sequence and allowed for reducing the limb swelling during the reperfusion phase.

\section{Donor Limb Preparation}

Incisions were given on the mid-flexor and mid-extensor surface, from the distal arm extending up to the mid-forearm level. Biceps brachii, brachialis, and triceps were identified and tagged; each one of these muscles was followed till their insertion individually and the entire muscle bulk was totally removed, leaving only the tendinous portion with its bony insertion behind.

Brachial artery with its venae commitantes was gently dissected only to allow enough mobility for the anastomosis with the recipient artery and veins. Basilic vein and cephalic vein were identified and tagged in the distal arm level and dissected from the skin flaps for a few centimeters to allow for enhanced mobility in relation to the skin flaps and straightline anastomosis. The radial nerve was dissected between the brachialis and brachioradialis muscles and its individual branches to the brachioradialis and ECRL and extensor carpi radialis brevis were identified and isolated.

Radial nerve dissection was done till identification of superficial and deep branch, which were individually tagged for separate anastomosis. Median nerve was traced up to just proximal to the elbow crease and the ulnar nerve was dissected up to just before its entry in to the cubital tunnel. All identified structures were individually tagged ( - Fig. 12) showing muscles and nerves dissected and tagged individually in donor limb and (-Fig. 13) showing completed dissection of the right-side donor upper limb).

During the dissection, it is mandatory to carefully look for small vessels and do a very careful preemptive hemostasis; this would eventually determine the smooth postoperative recovery of the patient by minimizing the blood loss.

\section{Transplantation Surgery}

After preparation of both donor and recipient limbs, bone fixation was done first, As there was a gross discrepancy in the cross-section of both the donor and recipient bones, decision was taken to match only the lateral cortical surfaces of the bones and use a single, $4.5 \mathrm{~mm}$ LCDCP with four holes on each side (-Fig. 14) showing osteosynthesis performed on the right side). The lower end of the LCDCP was contoured according to the shape of lateral supracondylar ridge on the donor bone. The additional bone was discarded from the donor limb and the osteosynthesis performed. The osteosynthesis site was later covered with the elevated periosteal flaps. Three major muscles were repaired first so as not to disturb the arterial and nerve anastomosis later.

First, the recipient brachialis muscle was sutured to the brachialis tendon of the donor with Pulvertaft weave technique; using number one Prolene sutures, the repair was executed, stretching the muscle in maximum tension and keeping the elbow flexed at 90 degrees. After the repair, the extra length of the donor tendon was folded upon itself and further utilized to reinforce the suture site and the remaining length was discarded. Similar technique was adopted for biceps tendon repair, with the elbow in nearly 90 degrees of

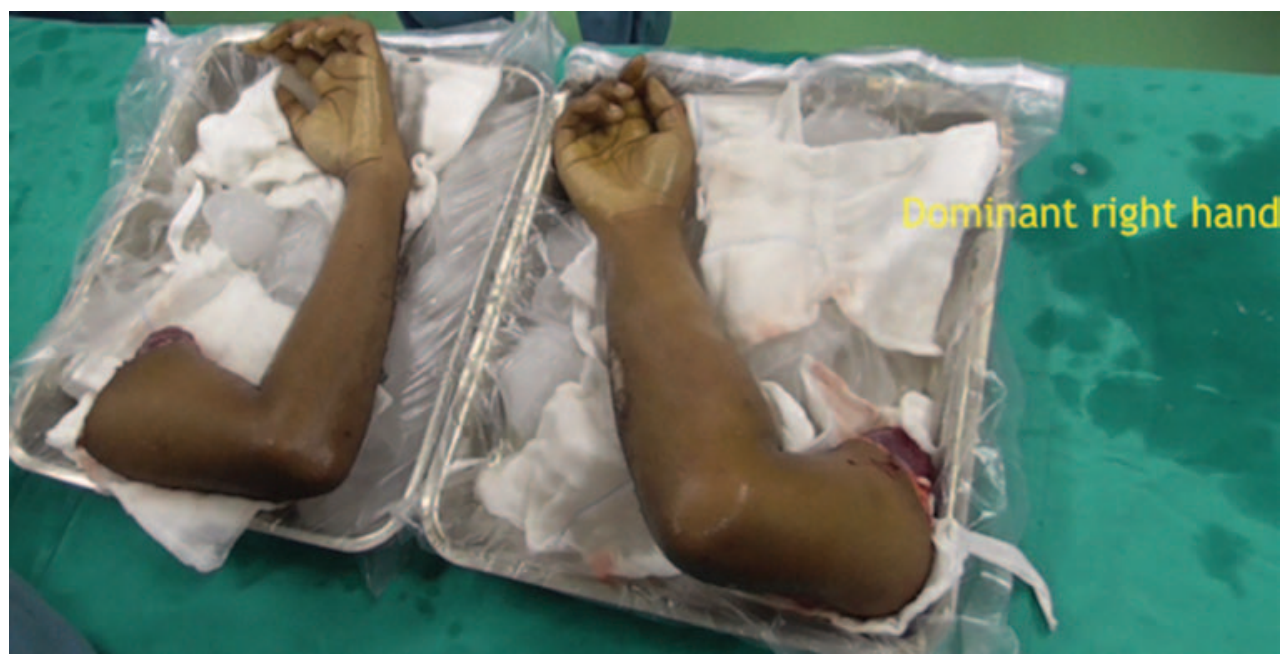

Fig. 11 The retrieved donor upper limbs. 


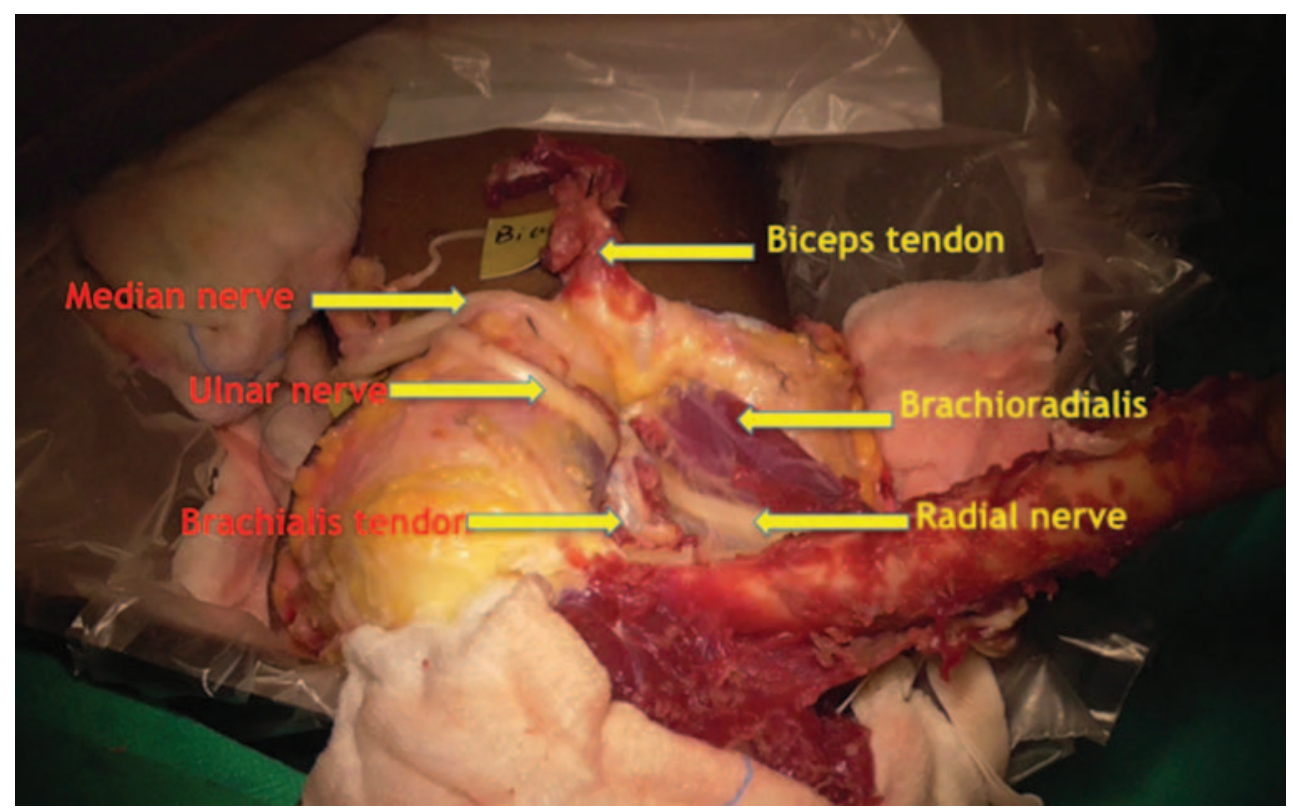

Fig. 12 Muscles and nerves dissected and tagged individually in donor limb.

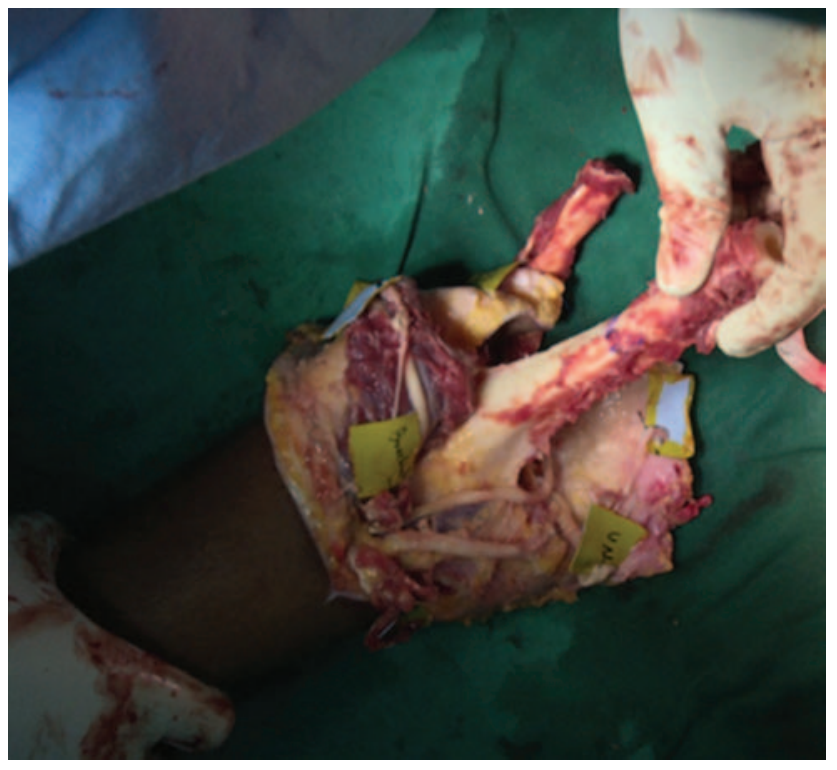

Fig. 13 Completed dissection of the right-side donor upper limb. flexion and muscle in maximum tension (-Fig. 15) showing biceps muscle Pulvertaft weave with donor biceps tendon with elbow in 90-degree flexion and (-Fig. 16) showing completed muscle repair). Thereafter, the initial weaving of triceps was done with elbow in nearly full extension and muscle in maximum tension.

Brachial artery was anastomosed next; as there was a big discrepancy in size of the vessels, with donor vessel being larger, fish mouthing of the recipient vessel was required for matching the diameter and repair was done with 8/0 Nylon suture. One of the venae comitantes was anastomosed at this time and the vascular clamps were released, perfusing the limbs (-Fig. 17) showing completed brachial artery and venae comitantes anastomosis).

Intravenous MP 500 mg was given just before and immediately after clamp release.

After revascularization, triceps muscle repair was completed by placing the donor tendinous part onto the recipient musculotendinous region and weaving them together using 1-0 Prolene. On the right side, the donor brachioradialis

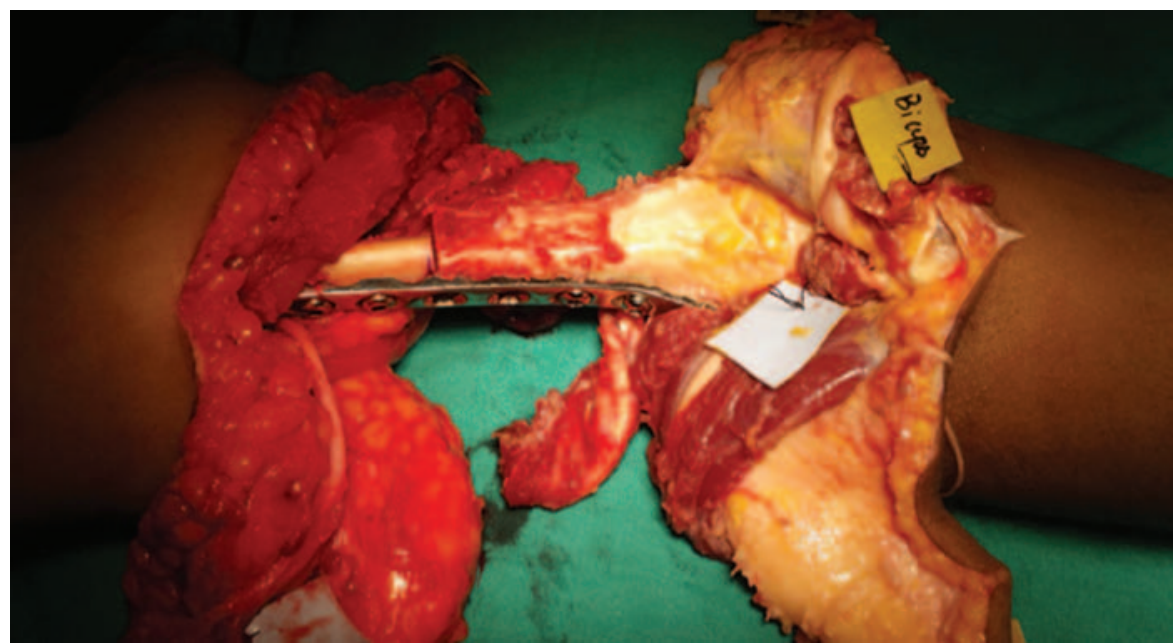

Fig. 14 Osteosynthesis performed on the right side. 


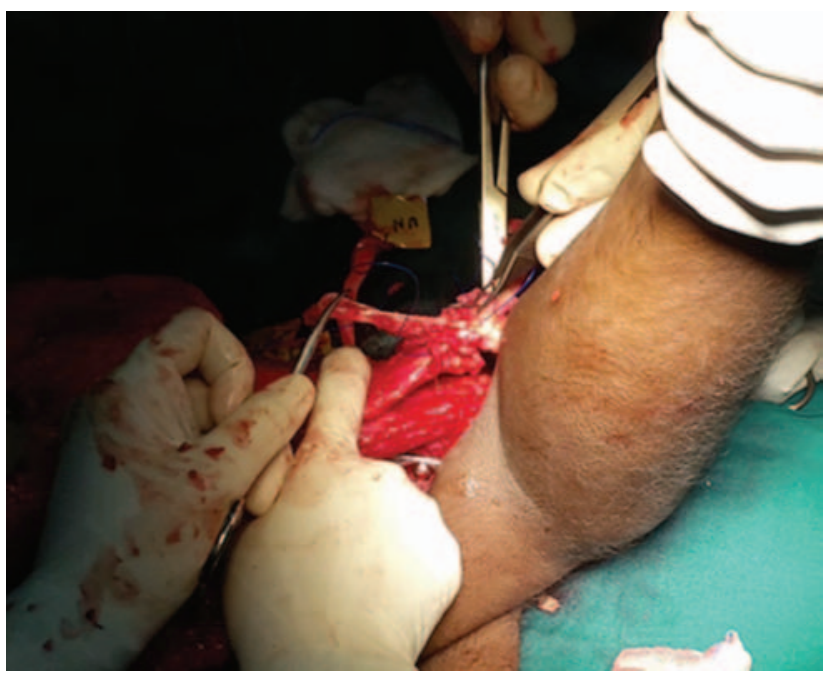

Fig. 15 Biceps muscle Pulvertaft weave with donor biceps tendon with elbow in $90^{\circ}$ flexion.

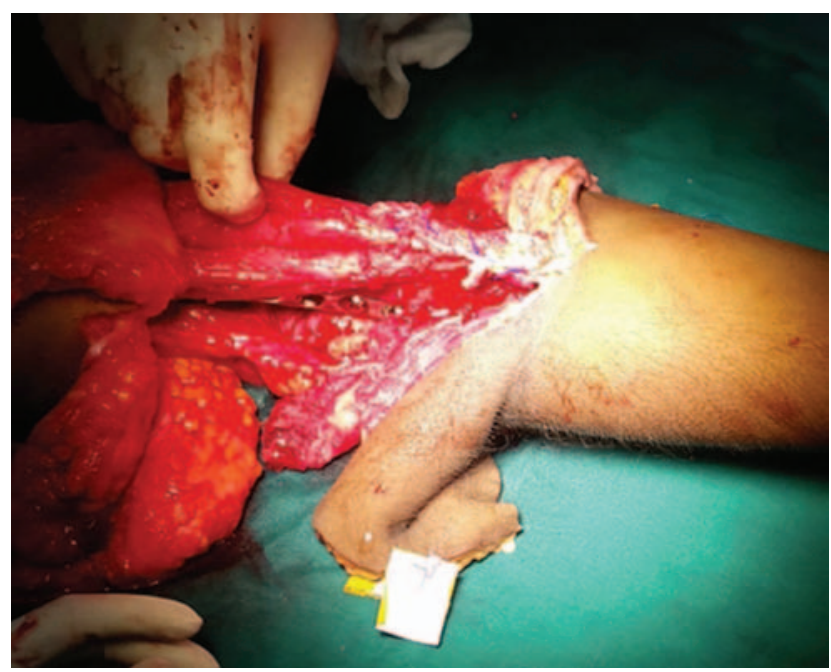

Fig. 16 Completed muscle repair. muscle was sutured to the humerus periosteum and its motor nerve anastomosed to the corresponding branch of the radial nerve entering the muscle, but on the left side as the recipient had a longer available length of the brachioradialis muscle, it was superimposed on to the donor brachioradialis muscle and sutured using No 2 Vicryl.

The motor nerve to ECRL from the recipient was anastomosed to the respective motor branch from the donor. After restoring the circulation, one more venae commitantes and basilic and cephalic veins were anastomosed using 8/0 nylon suture. In the median and ulnar nerve, an epineural repair was done using 9/0 Nylon sutures that was reinforced with tissue glue. The superficial and deep branches of the radial nerve were anastomosed separately using 10/0 Nylon sutures. Finally, the lateral antebrachial and medial antebrachial cutaneous nerve coaptation was done with 10/0 Nylon suture. All the nerve coaptations were also secured with a fibrin glue (EVICEL). The donor skin flaps were trimmed, interdigitated, and sutured in two layers using Vicryl 2-0 and Nylon 3-0. One number 14 closed suction drain was placed posteriorly and

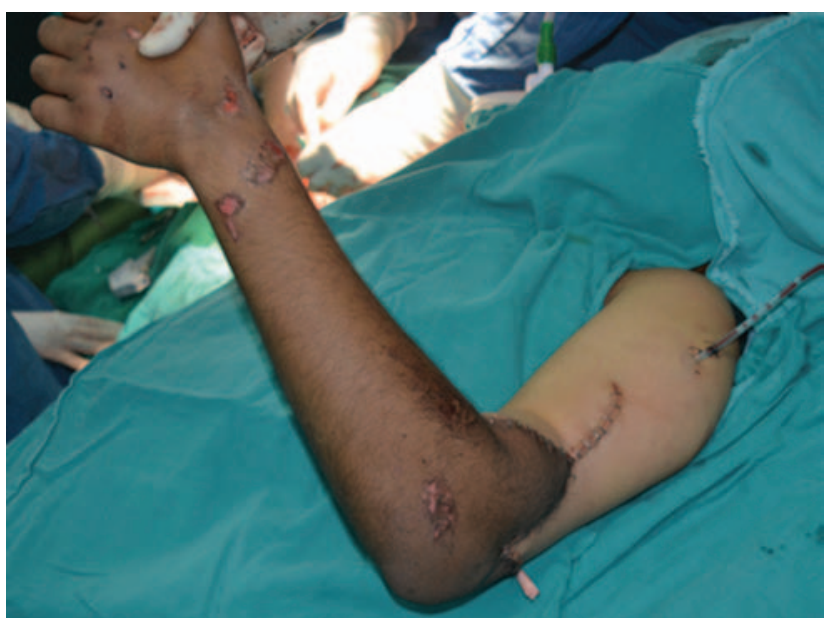

Fig. 18 Completed transplant with interdigitated skin flaps.

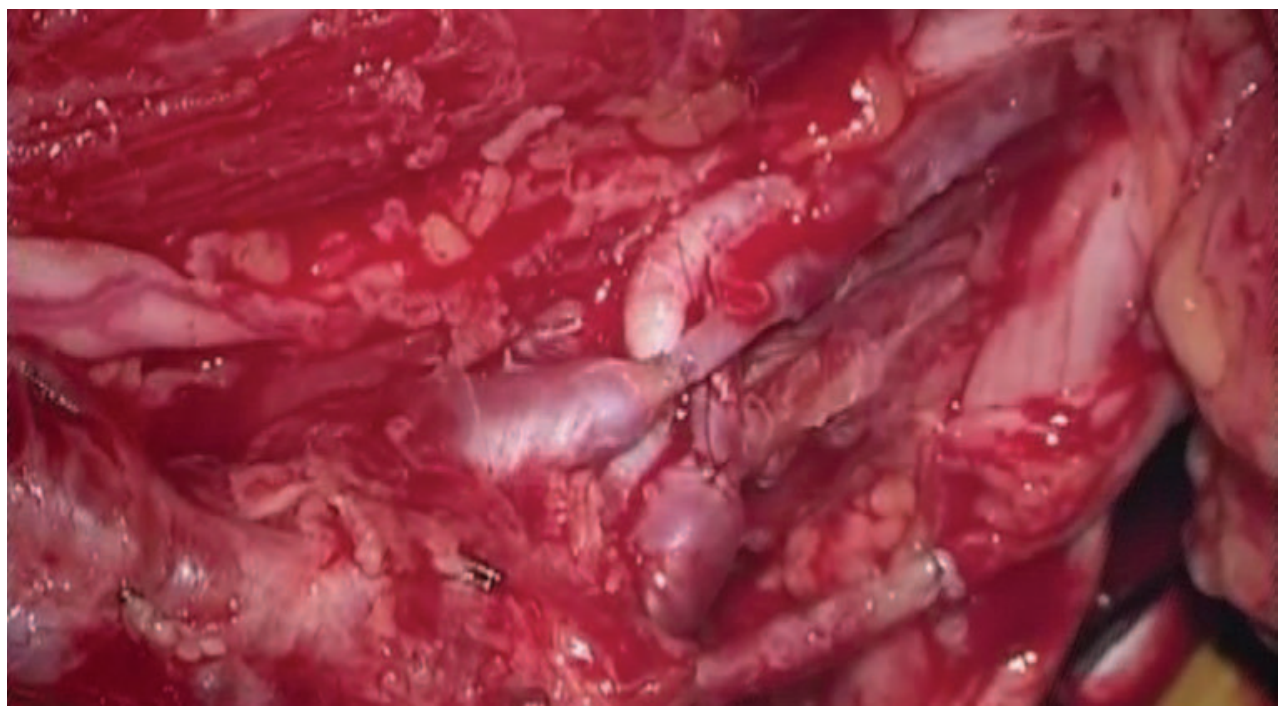

Fig. 17 Completed brachial artery and venae comitantes anastomosis. 
two glove drains were placed anteriorly (-Fig. 18) showing completed transplant with interdigitated skin flaps).

Following this the perfusion of the limb was rechecked that was found to be good. At the end of the procedure, supraclavicular blocks were administered bilaterally for pain relief and vasodilatation. Gamgee pads were used to cover the suture sites. Both the upper limbs were supported on two pillows to maintain the elevation; no splintage was given.

During the procedure, $9 \mathrm{~L}$ of crystalloid and 1 and half $\mathrm{L}$ of colloid were transfused along with that six units of packed red blood cells and four units of fresh frozen plasma were transfused

\section{Postoperative Monitoring and Care}

Patient was nursed in isolation transplant intensive care unit as per protocol. The limb vascularity was monitored using the pulse-oximeter probes attached on either hand and connected to separate monitors, along with one monitor connected to the great toe as a control. Monitoring of the radial and ulnar flow was done periodically using hand-held Doppler probes. The immunosuppression was monitored by a dedicated transplant immunology and nephrology team. The patient was mobilized by third postoperative day, using a specially devised walker trolley with supportive splint for hands (-Fig. 19) showing custom-made walking trolley for early patient mobilization).

The right limb of the donor had one radial arterial line placed, when he was on a ventilator; as a result some intimal damage might have been there, resulting in a localized block in the flow of right radial artery, and for the initial 2 days the right hand was perfusing on the ulnar artery alone.

Prostaglandin E1 (PGE1) infusion was started intravenously with concentration of $500 \mu \mathrm{g} / 50 \mathrm{~mL}$ normal saline diluted to $200 \mathrm{~mL}$ administered at the rate of $1 \mathrm{~mL} / \mathrm{min}$ for a period of

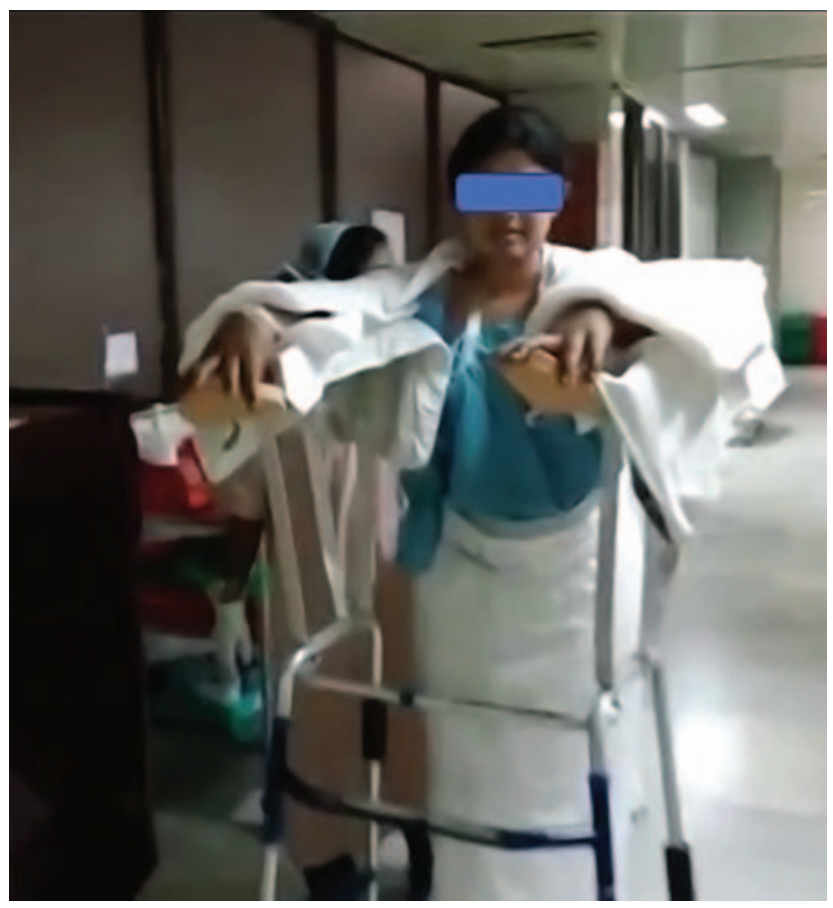

Fig. 19 Custom-made walking trolley for early patient mobilization.
2 days postoperatively, and by the third postoperative day the radial pulse was detectable. The donor right hand had multiple deep abrasions that healed over the ensuing 3 weeks period by daily dressing with local antibiotic ointment.

\section{Results}

Patient was in the transplant intensive care unit for a period of 14 days after which she was shifted out to an isolation ward. Her postoperative recovery was uneventful henceforth. The total duration of hospitalization was 30 days. Her functional recovery was also satisfactory. At the end of 1 year, her DASH score came down to 24 from the preoperative value of 68.9 , indicating significant reduction in her perceived disability.

\section{Discussion}

It is a known fact that the patients suffering from aboveelbow amputation perform poorly during post-amputation rehabilitation both physically and mentally. They seldom adapt well with the prosthesis. Hence, it proves to be a severely debilitating condition because they are completely dependent, even for basic sanitary care and suffer from a poor quality of life. ${ }^{12,13}$ Even though there have been doubts regarding the functional recovery after an arm-level/transhumeral transplant, because of the above-mentioned factors, transplantation is still considered to be an acceptable solution for high upper limb level amputees. ${ }^{14}$

From a technical point of view, there are certain advantages with the arm-level transplants such as:

1) Larger diameter of vessels,

2) Only one bone for osteosynthesis and

3) Lesser number of structures to be repaired compared with the forearm level transplants. ${ }^{10,15}$

In this case, the donor was a young male and the recipient was a young female (gender mismatch). And the length of radius and ulna present in the forearm stump of the recipient was not adequate to perform any kind of osteosynthesis; hence, a supracondylar transplant was performed. The challenges that we faced in this case were as follows:

1. Discrepancy in the vessel circumference.

2. Discrepancy in the size of the humerus, due to which only lateral surface was matched and osteosynthesis was performed using a single LCDCP.

3. Larger bulk of muscles was transplanted; hence, it was important to keep the ischemia time as short as possible.

4. Due to the presence of neuromas, the median nerve on the right side was resected at a level $10 \mathrm{~cm}$ higher than the left side and similarly the scar and fibrosis at the site of right radial nerve caused it to be excised $10 \mathrm{~cm}$ higher than the left sided radial nerve that would eventually delay the return of function in the right upper limb.

The use of PGE1 infusion has been described to be of benefit in patients with critical limb ischemia secondary to peripheral vascular occlusive disease. PGE1 agonist binds to the PGE receptors causing corporal smooth muscle relaxation that in 


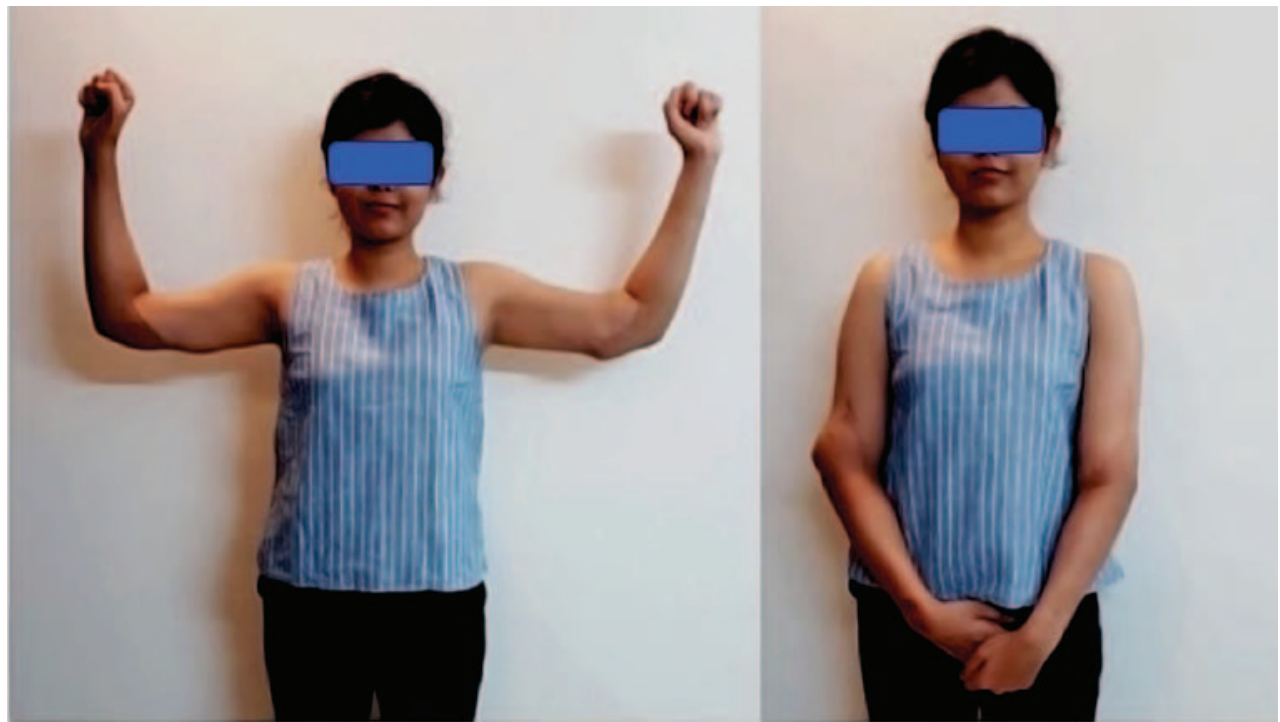

Fig. 20 Pictures of the patient at 20 months postoperatively.

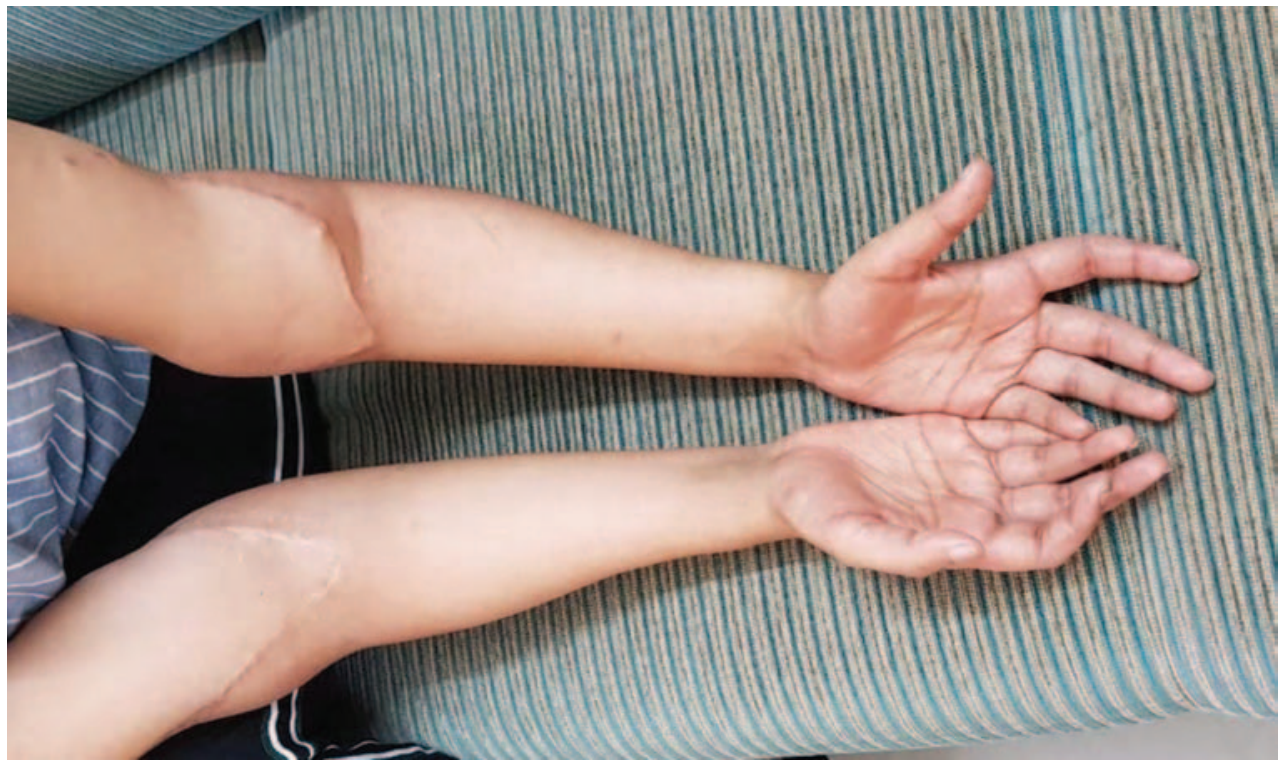

Fig. 21 Pictures of the patient at 20 months postoperatively.

turn leads to increase in peripheral blood flow by vasodilation and inhibits platelet aggregation. ${ }^{16}$ In our patient, due to previous cannulation of the radial artery, radial pulse on the right hand was not palpable initially for which PGE1 infusion was started; effective dose that was given to our patient was $2.5 \mu \mathrm{g} / \mathrm{min}$; the maximum safe dose is up to $10 \mu \mathrm{g} / \mathrm{min}$. The infusion was continued for 2 days, following which the radial pulse became palpable.

Till now the progress of the patient has been very satisfactory ( - Figs. 20 and $\mathbf{2 1}$ ) showing the postoperative pictures of the patient at 20 months), and a detailed report regarding the functional outcome would be presented in the next article.

\section{Conclusion}

Supracondylar level arm-transplantation requires a highly coordinated team effort with precise preoperative planning, along with meticulous attention to detail to achieve a successful outcome. In properly selected patients, it could be a life-changing procedure, worth all the effort.

\section{Conflict of Interest}

None declared.

\section{References}

1 Siemionow MZ, Kulahci Y, Bozkurt M. Composite tissue allotransplantation. Plast Reconstr Surg 2009;124 (6, Suppl):e327-e339

2 Shores JT, Higgins JP, Lee WP. Above-elbow (supracondylar) arm transplantation: clinical considerations and surgical technique. Tech Hand Up Extrem Surg 2013;17(4):221-227

3 Petruzzo P, Dubernard JM. The International Registry on Hand and Composite Tissue allotransplantation. Clin Transpl $2011 ; \cdots \cdot \cdots: 247-253$

4 Murphy BD, Zuker RM, Borschel GH. Vascularized composite allotransplantation: an update on medical and surgical 
progress and remaining challenges. J Plast Reconstr Aesthet Surg 2013;66(11):1449-1455

5 Jones NF, Schneeberger S. Arm transplantation: prospects and visions. Transplant Proc 2009;41(2):476-480

6 Messner F, Grahammer J, Hautz T, Brandacher G, Schneeberger S. Ischemia/reperfusion injury in vascularized tissue allotransplantation: tissue damage and clinical relevance. Curr Opin Organ Transplant 2016;21(5):503-509

7 Tuffs A. Munich surgeons perform world's first transplantation of whole arms. BMJ 2008;337:a1162

8 Iglesias M, Salazar-Hernandez F, et al. Anatomical and microsurgical implications in total and midarm transplantation. J Reconstr Microsurg Open 2017;2:e94-e102

9 Cavadas PC, Ibáñez J, Thione A, Alfaro L. Bilateral transhumeral arm transplantation: result at 2 years. Am J Transplant 2011;11(5):1085-1090

10 Jablecki J, Kaczmarzyk L, Domanasiewicz A, et al. Result of arm-level upper-limb transplantation in two recipients at 19- and 30-month follow-up. Ann Transplant 2012;17 (3):126-132
11 Chhabra A, Ahlawat S, Belzberg A, Andreseik G. Peripheral nerve injury grading simplified on MR neurography: as referenced to Seddon and Sunderland classifications. Indian J Radiol Imaging 2014;24(3):217-224

12 Datta D, Selvarajah K, Davey N. Functional outcome of patients with proximal upper limb deficiency-acquired and congenital. Clin Rehabil 2004;18(2):172-177

13 Amadio PC. Outcome assessment in hand surgery and hand therapy: an update. J Hand Ther 2001;14(2):63-67

14 Gorantla VS, Brandacher G, Schneeberger S, et al. Favoring the risk-benefit balance for upper extremity transplantation-the Pittsburgh Protocol. Hand Clin 2011;27(4):511-520, ix-X

15 Hartzell TL, Benhaim P, Imbriglia JE, et al. Surgical and technical aspects of hand transplantation: is it just another replant? Hand Clin 2011;27(4):521-530, x

16 Weiss T. [Mechanisms of action of prostaglandin E1 in therapy of peripheral arterial occlusive diseases]. Vasa 2003;32(4):187-192 